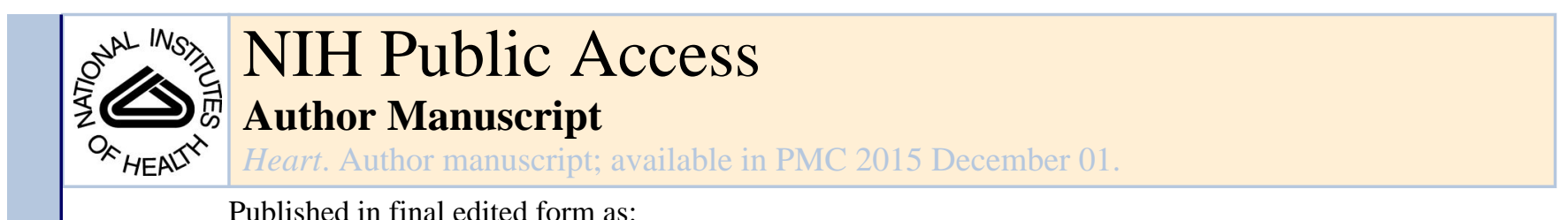

Published in final edited form as:

Heart. 2014 December 1; 100(23): 1871-1877. doi:10.1136/heartjnl-2014-306067.

\title{
Periprocedural imaging and outcomes after catheter ablation of atrial fibrillation
}

\author{
Benjamin A Steinberg ${ }^{1,2}$, Bradley G Hammill ${ }^{2}$, James P Daubert ${ }^{1,2}$, Tristram D Bahnson ${ }^{1,2}$, \\ Pamela S Douglas $^{1,2}$, Laura G Qualls ${ }^{2}$, Sean D Pokorney ${ }^{1,2}$, Hugh Calkins ${ }^{3}$, Lesley H \\ Curtis $^{2}$, and Jonathan P Piccini ${ }^{1,2}$ \\ ${ }^{1}$ Duke Center for Atrial Fibrillation, Duke University Medical Center, Durham, North Carolina, USA \\ ${ }^{2}$ Duke Clinical Research Institute, Durham, North Carolina, USA \\ ${ }^{3}$ Johns Hopkins Medical Institutions, Baltimore, Maryland, USA
}

\begin{abstract}
Objective-Catheter ablation of atrial fibrillation (AF) has become an increasingly safe and effective therapy. This has been partly attributed to the use of adjunctive imaging modalities. We aimed to describe the use and associated outcomes of periprocedural imaging for AF ablation.

Methods-We studied all Medicare fee-for-service claims for AF ablation from July 2007 to December 2009, and identified associated imaging studies before and during ablation, including transoesophageal echocardiography (TEE), intracardiac echocardiography (ICE), CT and MRI. The primary outcomes were death, stroke or transient ischaemic attack (TIA), repeat ablation, and bleeding (pericardial or vascular) at 6 months.
\end{abstract}

Results-11 525 patients underwent AF ablation during the study period. There was significant variation in imaging use at the practice level. In addition to electroanatomic mapping, 53\% $(\mathrm{n}=6060 / 11525)$ underwent TEE, 67\% $(\mathrm{n}=7715 / 11525)$ received ICE, and 50\% $(\mathrm{n}=5724 / 11525)$

Copyright Article author (or their employer) 2014. Produced by BMJ Publishing Group Ltd (\& BCS) under licence

Correspondence to Dr Jonathan P Piccini, Electrophysiology Section, Duke Center for Atrial Fibrillation, Duke University Medical Center, Duke Clinical Research Institute, PO Box 17969, Durham, NC 27710, USA; jonathan.piccini@duke.edu.

Additional material is published online only. To view please visit the journal online (http://dx.doi.org/10.1136/heartjnl-2014-306067). Contributors Conception and design: BAS, BGH, LGQ, LHC, JPP. Planning and conduct: BAS, BGH, LGQ, SDP, HC, LHC, JPP. Interpretation of data: BAS, JPD, TDB, PSD, HC, LHC, JPP. Reporting and critical revisions of manuscript: BAS, BGH, JPD, TDB, PSD, SDP, HC, LHC, JPP.

Competing interests JPD reported receiving research funding from Biosense Webster, Biotronik, Gilead and Medtronic; honoraria from Biotronik, Boston Scientific, Medtronic, Sorin and St. Jude; serving as an expert witness for a patient with sudden cardiac death due to coronary artery disease; having ownership interest in Biosense Webster inherited by his wife/children in a generation-skipping trust for the children; and receiving consultant/advisory board income from Premier. TDB reported receiving research funds from Medtronic and St. Jude Medical and serving as a consultant and/or speaker for Boehringer Ingelheim, ChanRX, Sequel Pharma and Sanofi-Aventis. PSD reported receiving research funding from Atritech, Edwards Lifesciences, Ikaria and Miracor Medical Systems; serving as a consultant for UptoDate/Kluwer, Translation Research in Oncology and CardioDX; and having equity in CardioDX and Universal Oncology. SDP reported modest consulting/advisory board support from Janssen Pharmaceuticals and modest research support from AstraZeneca and Boston Scientific. LHC reported receiving research funding from GlaxoSmithKline, Janssen and Novartis. JPP reported receiving grants for clinical research from ARCA biopharma, Boston Scientific, Janssen Pharmaceuticals and Resmed; and serving as a consultant to Forest Laboratories, Janssen Pharmaceuticals and Spectranetics. LHC and JPP have made available online detailed listings of financial disclosures (https://www.dcri.org/about-us/conflict-of-interest). No other authors reported financial disclosures.

Provenance and peer review Not commissioned; externally peer reviewed. 
underwent a preprocedure CT or MRI. Imaging generally increased from 2007 to 2009. After adjustment, the use of preablation CT or MRI was associated with a significantly lower risk of stroke or TIA ( $0.4 \%$ vs $0.9 \%$, adjusted HR $0.46,95 \%$ CI 0.28 to $0.74, \mathrm{p}=0.002)$, and the use of ICE was associated with a lower risk of repeat ablation (5.7\% vs $8.5 \%$, adjusted HR $0.59,95 \%$ CI 0.37 to $0.92, \mathrm{p}=0.02)$ but higher risk of bleeding ( $1.1 \%$ vs $0.7 \%$, adjusted HR $1.76,95 \%$ CI 1.15 to $2.70, \mathrm{p}=0.009$ ).

Conclusions-Periprocedural imaging for AF ablation is increasingly used, although practice patterns vary widely. Our data suggest that periprocedural imaging is associated with better outcomes after catheter ablation; however, prospective studies of periprocedural imaging strategies are warranted.

\section{INTRODUCTION}

Catheter ablation has been shown to be superior to antiarrhythmic therapy for the treatment of drug refractory, symptomatic atrial fibrillation (AF). ${ }^{1}$ The past decade has produced significant advances in catheter ablation approaches for AF and improved procedural outcomes. This may be due to a variety of factors, including better patient selection, technological advances and greater operator experience. ${ }^{2}$ In particular, cardiovascular imaging before and during $\mathrm{AF}$ ablation has become integral to the procedure. ${ }^{3} \mathrm{CT}$ and/or MRI may be used to define anatomy prior to the ablation; transoesophageal echocardiography (TEE) is often used to exclude the presence of left atrial appendage (LAA) thrombus; and intracardiac echocardiography (ICE) and/or electroanatomic mapping (EAM) can be used for spatial guidance during the procedure.

The extent to which adjunctive imaging is employed to guide AF ablation in practice is poorly described; the largest studies include international surveys of high-volume centres that are not representative of community practice. ${ }^{2}$ Furthermore, there is little evidence to demonstrate improved outcomes with any specific periprocedural imaging strategy. ${ }^{4-6}$ Therefore, the objectives of the current analyses were to: (1) describe the contemporary use of periprocedural imaging technologies in a broad cohort of patients undergoing ablation for $\mathrm{AF}$ and (2) describe the association between periprocedural imaging use and outcomes. More specifically, we hypothesised that the use of periprocedural imaging would be associated with better outcomes postprocedure, including repeat ablation.

\section{METHODS}

\section{Data sources}

We used inpatient, outpatient and carrier claim files, as well as the corresponding denominator files from the US Centers for Medicare and Medicaid Services for all Medicare beneficiaries who underwent catheter ablation between 1 July 2007 and 31 December 2009. Medicare inpatient files included facility-based claims associated with services provided to hospitalised patients while outpatient files included facility-based claims associated with non-hospitalised patients. Claims for provider and other professional services, across all settings, were found in the carrier files. Demographic data, enrolment information and eligibility, as well as dates of death were found in the denominator files. The current 
analysis was limited to beneficiaries $\ 65$ years of age who were continuously enrolled in the Medicare fee-for-service programme for at least 6 months prior to the index procedure (to assess comorbid conditions).

\section{Study population}

Eligible patients were identified consistent with previously described methods, ${ }^{7}$ by searching carrier files for claims for intracardiac ablation of supraventricular tachycardia (Healthcare Common Procedure Coding System (HCPCS) code 93651) having an associated primary diagnosis code for AF (International Classification of Diseases, Ninth Revision, Clinical Modification (ICD-9-CM) diagnosis code 427.31). To improve specificity, we excluded patients who did not receive EAM, who underwent atrioventricular node ablation (HCPCS 93650), and those with secondary diagnoses of anomalous atrioventricular excitation (ICD-9-CM 426.7) or paroxysmal supraventricular tachycardia (ICD-9-CM 427.0). For patients with multiple ablations, we defined the earliest as the index procedure.

\section{Patient characteristics}

Comorbid conditions were identified using diagnosis codes from claims during the 180 days preceding the ablation procedure. Previously-validated coding algorithms by Quan et al and Birman-Deych et al were used to identify diabetes, ischaemic heart disease, peripheral vascular disease, heart failure, hypertension, chronic pulmonary disease, chronic kidney disease, dementia, cancer, valvular heart disease, and prior stroke or transient ischaemic attack (TIA) ${ }^{89}$ Atrial flutter was identified by ICD-9-CM code 427.32. International normalised ratio testing was identified using CPT code 85610.

\section{Imaging modalities}

Imaging tests were identified in carrier claims based on HCPCS codes (see online supplementary material). We searched for preablation TEE, chest/cardiac CT and chest/ cardiac MRI in the 4 weeks prior to the ablation date and intraprocedural ICE on the same date as the procedure. We assumed that all TEEs performed on the day of the ablation were done prior to the ablation procedure. For each test observed, we recorded the date of the imaging most proximate to the index date for the purposes of summarising timing of each modality.

\section{Outcomes}

Prespecified, primary outcomes events included death, stroke/TIA,${ }^{10}$ haemopericardium or cardiac tamponade, heart failure, vascular complications requiring surgery, ${ }^{11}$ and repeat ablation within 6 months. Coding definitions for these events are given in the online supplementary material. Some events were combined for analysis due to very low event rates.

\section{Statistical methods}

To describe the periprocedural imaging received by the study population, we presented the number and per cent of patients receiving each imaging procedure, overall and by subgroups 
defined by year, age $(<75, \geq 75), \mathrm{CHADS}_{2}$ score $(0-1, \geq 2)$ and presence of heart failure. $\chi^{2}$ Tests were used to assess differences within subgroups. Practice-level variability in imaging utilisation was also presented using histograms. Practices were defined by 5-digit ZIP code of the physician performing the ablation. We limited practice-level results to those with five or more ablations in the study cohort.

We described baseline characteristics by periprocedural imaging received. Patients were categorised into four groups based on the combination of periprocedural TEE and CT/MRI they received. Continuous data were presented as means with SDs and categorical data as frequencies with percentages. Differences were assessed using Kruskal-Wallis tests for continuous variables and $\chi^{2}$ tests for categorical variables.

Observed event rates were presented by periprocedure imaging group. All event and imaging rates were estimated using the cumulative incidence function, which accounts for censoring and competing risks, and differences between groups were assessed using Gray tests. In this study, death and repeat ablation were treated as competing risks for each other and for all other clinical and imaging events. Data for all patients not experiencing death or repeat ablation were censored when they enrolled in Medicare managed care or at the end of the follow-up period (31 December 2009).

In order to test our hypotheses, we used proportional hazards regression models to estimate the association of periprocedural imaging with clinical outcomes. We compared adjusted outcomes according to the use versus non-use of a given modality, regardless of the other imaging modalities used in a given patient. In addition to indicators for periprocedural TEE, CT/MRI and ICE, all models included patient demographics, baseline characteristics, procedure year and inpatient status. We accounted for both provider variation in care delivery and clustering of patients within providers by including provider-level random intercepts in all models.

In order to assess the validity of the study population and the robustness of the findings, we performed subsequent sensitivity analyses: (1) excluding patients with a diagnosis of atrial flutter and (2) using different follow-up periods for the endpoints (bleeding within 30 days, stroke/TIA within 90 days and repeat ablation at 1 year).

All statistical analyses were performed using SAS software (V.9.3, SAS Institute, Cary, North Carolina, USA).

\section{RESULTS}

We identified 11525 patients with qualifying AF ablations between 1 July 2007 and 31 December 2009. Rates of periprocedural imaging, overall and by subgroup, are shown in table 1. CT/MRI was used in 50\% of ablations, and TEE was used in 53\%. Older patients ( $\geq 75$ ), those with $\mathrm{CHADS}_{2}$ scores $\geq 2$ and patients with heart failure had significantly lower $\mathrm{CT} / \mathrm{MRI}$ and ICE use ( $\mathrm{p}<0.001$ for each). TEE use was higher in younger patients (54\% vs $49 \%, \mathrm{p}<0.001)$ and those with heart failure ( $54 \%$ vs $52 \%$ without heart failure, $\mathrm{p}=0.02$ ), however was not different by $\mathrm{CHADS}_{2}$ score ( $53 \%$ for $0-1$ vs $52 \%$ for $\geq 2, \mathrm{p}=0.3$ ). 
Timing of preprocedural imaging is shown in figure 1; TEE was performed closest to ablation (mean 1.2 days prior), with CT and MRI approximately 1 week prior (mean 7.9 and 7.0, respectively). Distribution of periprocedural imaging utilisation, by practice, is shown in figure 2. Use of ICE (median 58.8\%, IQR 20.0\%-82.8\%), TEE (median 46.9\%, IQR 20.0\%-72.1\%) and CT/MRI (median 42.9\%, IQR 16.7\%-62.5\%) varied across practices.

Patients were subsequently grouped according to preprocedural imaging utilisation: EAM only, EAM with TEE, EAM with CT/MRI, and EAM with TEE and CT/MRI. ICE was measured independently in each of these groups. Baseline characteristics and ICE use in each group are shown in table 2 . There were differences in patient characteristics across imaging strategies. Patients receiving both TEE and CT/MRI were modestly younger (mean 71 vs 72.4 years for EAM alone), but less likely to have a history of atrial flutter (33\% vs $43 \%$ ) or chronic kidney disease (5.8\% vs $8.6 \%$ ). Use of oral anticoagulation increased with additional imaging (within 30 days prior, from $76 \%$ for EAM only to $84 \%$ for EAM with TEE and CT/MRI).

Use of periprocedural imaging, by year, is shown in figure 3. Rates increased from 2007 to 2009 for TEE, cardiac CT and ICE ( $p_{\text {trend }}<0.001$ for each).

\section{Specific imaging modalities and clinical outcomes}

Unadjusted rates for each outcome are shown in table 3; 6-month mortality following ablation ranged from $1.0 \%$ for patients receiving all available imaging modalities to $1.9 \%$ for patients undergoing a procedure with only EAM $(\mathrm{p}=0.03$ for comparison across all groups). Rates of postprocedure stroke/TIA were low overall (range $0.4 \%-1.0 \%, \mathrm{p}=0.009$ across imaging groups). Adjusted outcomes are show in table 4 . None of the imaging modalities was associated with differential 6-month mortality.

TEE

Overall, 53\% ( $\mathrm{n}=6060 / 11525)$ of patients undergoing ablation had a preprocedure TEE. Patients $\geq 75$ years of age were less likely to undergo preprocedure TEE (44\% vs $52 \%$ ).

Paradoxically, patients at higher risk for stroke were less likely to have a preprocedure TEE: $52 \%$ in those with a $\mathrm{CHADS}_{2}$ score of $0-1$ versus $48 \%$ in those with a $\mathrm{CHADS}_{2}$ score $\geq 2$. After adjustment, the use of TEE was not associated with a decreased risk of TIA or stroke ( $0.6 \%$ vs $0.7 \%$, adjusted HR 0.94 ( $95 \%$ CI 0.58 to 1.53 ), $\mathrm{p}=0.80$ ).

ICE

Overall, 67\% ( $\mathrm{n}=7715 / 11525)$ of ablations incorporated ICE. ICE was more often employed in those without heart failure (68\% vs 64\%) and in those with $\mathrm{CHADS}_{2}$ scores of 0-1 (71\% vs $64 \%)$. After adjustment, the use of ICE was associated with a higher risk of severe bleeding ( $1.1 \%$ vs $0.7 \%$, adjusted HR 1.78 (95\% CI 1.11 to 2.83 ), $\mathrm{p}=0.02$ ), and lower repeat ablation rates at 6 months ( $5.7 \%$ vs $8.5 \%$, adjusted HR 0.68 (95\% CI 0.57 to 0.82 ), $\mathrm{p}<0.001)$. 


\section{Preprocedure CT and MRI}

Only 50\% ( $\mathrm{n}=5724 / 11525$ ) received a preprocedure CT/MRI. The use of CT/MRI was more common in lower-risk patients, including by age, CHADS2 scores and heart failure status (table 1). The use of CT/MRI prior to ablation was significantly associated with lower rate of 6-month stroke/TIA ( $0.4 \%$ vs $0.9 \%$, adjusted HR 0.46 (95\% CI 0.27 to 0.78 ), p=0.004).

\section{Sensitivity analyses}

Results of a sensitivity analysis excluding patients with any diagnosis of atrial flutter were consistent with the primary study population. Sensitivity analyses of endpoints at different follow-up periods yielded results qualitatively similar to the primary analysis, with limited power (see online supplementary material).

\section{DISCUSSION}

These data represent the largest patient-level description of adjunctive imaging strategies in catheter ablation of AF. There are several important findings from our analysis. First, multiple imaging modalities were used for AF ablation, often in combination, and their use increased over time. Second, periprocedural imaging utilisation varied significantly by operator. However, despite these differences, adverse outcomes were uncommon across this elderly cohort of patients undergoing catheter ablation. Finally, exploratory analyses of periprocedural imaging modalities demonstrated that the use of adjunctive imaging modalities may be associated with better outcomes.

The practice-level variability observed in this nationwide cohort reflects the dynamic landscape of AF ablation. Patients undergoing ablation include those with and without structural heart disease, as well as patients with varying degrees of AF severity (ie, paroxysmal vs persistent forms), and these differences may yield diverse ablative approaches. Additionally, ablative and mapping technologies continue to evolve, which may lead to increased use of adjunctive imaging (eg, CT/MRI guided registration). Most importantly, there are no randomised data demonstrating the superiority of any particular imaging strategy for patients undergoing AF ablation, likely contributing to variation in local practice. Recently, the European Heart Rhythm Association convened a policy conference to guide the use of, recommendations for and research priorities in cardiovascular imaging for electrophysiology procedures. ${ }^{3}$ While these recommendations support the use of imaging technologies to guide specific aspects of a variety of procedures, they acknowledge the shortcomings of these technologies, as well as the wide evidence gaps in their optimal implementation. The large variation in practice patterns in our data support the need for additional evidence on optimal periprocedural imaging strategies.

We also observed that patients at the highest risk (by age, $\mathrm{CHADS}_{2}$ score or heart failure status) had lower rates of imaging studies. This may be attributed to clinicians' hesitancy to expose higher-risk patients to more intensive therapies, yet even the non-invasive imaging modalities in our study were employed less commonly in higher-risk patients. Additional factors may be at play: for example, the characteristics of patients undergoing ablation may vary by provider or centre, with some centres both performing proportionally more 
procedures in higher-risk patients but having a practice pattern of not using adjunctive imaging modalities. We also cannot exclude payer influence in the decision to use adjunctive imaging. In the absence of robust clinical outcome data favouring one imaging strategy over another, the optimal application of these technologies to higher-risk populations is not clear.

Prior studies of outcomes related to imaging for AF ablation have been limited, often including a single modality or lacking patient-level data. ${ }^{24-6}$ The most recent results of a worldwide survey of ablation centres demonstrated no significant association between the use of EAM and subsequent freedom from AF. However, no other clinical outcomes were analysed, and data regarding other preprocedural and intraprocedural imaging modalities (TEE, CT, MRI, ICE) were not presented. These tests can help define left atrial anatomy, rule out the presence of thrombus and provide spatial guidance for catheter manipulation; our results yielded several important findings regarding the association between these imaging studies and clinical outcomes. It should be noted that all of these studies predate the availability of alternative ablative technologies that may influence the use of periprocedural imaging (ie, cryothermy balloon ablation).

The performance of a preprocedural TEE was not associated with clinical outcomes at 6 months-risk of stroke/TIA following ablation was similar in patients who underwent TEE compared with those who did not. Since our cohort only included patients undergoing ablation, it is likely that TEE appropriately selected out high-risk patients (ie, those with LAA thrombus) and, thus, the patients who subsequently underwent ablation represented the lowest risk among those who underwent TEE. Prior studies of TEE have identified thrombus in up to $3 \%-5 \%$ of patients referred for ablation. ${ }^{1213}$ Alternatively, it is also possible that the $\mathrm{CHADS}_{2}$ score (or equivalent historical markers) is a better discriminator for patients at high risk of stroke following ablation and TEE does not add significantly to risk stratification. Nevertheless, the use of TEE to reduce the risk of periprocedural stroke/TIA has not been formally evaluated in a prospective study despite recommendations by expert consensus. ${ }^{13}$

The use of adjunctive CT/MRI for image registration in addition to EAM has been shown to shorten procedure times and lead to increase rates of pulmonary vein isolation. ${ }^{1415}$ Adjunctive imaging and subsequent registration provide additional anatomic detail and may help avoid cardiovascular and structural complications. When we compared the use of CT/MRI with no CT/MRI (regardless of other imaging) after adjustment, we found that the use of preprocedural CT/MRI was associated with lower rates of postprocedure cardiovascular events. While these results are promising, they are also hypothesis-generating and require further study. CT and MRI are costly, often must be performed days in advance and volume-loading conditions may alter anatomy on the day of the procedure.

Alternatively, newer technology such as 3D rotational angiography or the use of ICEassisted EAM may provide effective and efficient anatomic assessment at the time of ablation. ${ }^{16}$

Last, we found a significant association between the use of ICE and increased risk of bleeding complications but lower repeat ablation rates. Use of ICE during the procedure 
requires additional and/or larger femoral venous access, which increases the risk of vascular complications. This may have also led to fewer subsequent ablations in those patients who experienced a complication. However, some providers employ ICE during radiofrequency applications to ensure appropriate contact between the ablation catheter and endocardium. Tissue contact force has been shown to correlate with extent of electrical isolation and subsequent clinical outcomes. ${ }^{1718}$ A recent analysis of ICE-assisted 3D mapping demonstrated shorter fluoroscopy times and shorter left atrial dwell times. ${ }^{19}$ While there were no differences in a small cohort with short term follow-up, these differences may translate into better outcomes when used in large populations. When considered in the context of smaller studies, our data raise the hypothesis that the use of ICE may lead to more durable pulmonary vein isolation and lower repeat ablation rates.

\section{Limitations}

This study used nationwide claims data. Accordingly, several potential limitations must be acknowledged when considering the results. They include coding and reporting biases.

Additionally, while our analyses employed multivariable adjustment, we cannot exclude the possibility of residual confounding or unmeasured confounding by covariates that may influence ablation outcome (eg, AF type). Although we used standardised and previously tested methods of identifying AF ablation, we cannot definitively exclude the capture of ablations for other arrhythmias. However, the results of sensitivity analyses of patients with and without a diagnosis of atrial flutter were consistent, demonstrating the robustness of our findings. These data could not identify prior AF ablations $>6$ months earlier or before 2007, and a proportion of index procedures in the current analysis may represent repeat ablations. Finally, all of the patients in our study were over the age of 65; these findings may have more limited applicability to younger patients undergoing AF ablation. Younger patients undergoing ablation are generally of lower risk and, thus, the differences in outcomes that we observed may be attenuated in such a population.

\section{CONCLUSIONS}

Use of periprocedure imaging modalities for $\mathrm{AF}$ ablation is increasing; however, there is significant variability in their use. Higher-risk patients appear to be less likely to undergo periprocedural imaging. Imaging with CT/MRI was associated with lower rates of stroke/ TIA, and ICE was associated with lower repeat ablation rates but a higher risk of bleeding. Overall, these data raise the hypothesis that periprocedural imaging is associated with better outcomes. Clinical trials are warranted to identify the optimal use and combination of periprocedural imaging modalities for AF ablation.

\section{Key messages}

\section{What is already known about this subject?}

Many providers use adjunctive imaging prior to and during AF ablation to risk-stratify and guide ablation.

\section{What might this study add?}


This study describes significant variability in use and outcomes of periprocedural imaging for AF ablation. The use of preprocedure CT or MRI was associated with a 54\% lower risk of stroke or TIA and the use of intracardiac ultrasound was associated with a $41 \%$ lower risk of need for repeat ablation but a $76 \%$ higher risk of bleeding.

\section{How might this impact on clinical practice?}

These data inform the decision to use adjunctive imaging technologies in patients undergoing AF ablation. They may also support the design of robust clinical trials, and the need for formal guidance.

\section{Supplementary Material}

Refer to Web version on PubMed Central for supplementary material.

\section{Acknowledgments}

Funding This study was funded by a grant from General Electric company acting through its GE healthcare business unit. Dr Steinberg was supported by grant T32H1710138 from the National Institutes of Health.

\section{REFERENCES}

1. Piccini JP, Lopes RD, Kong MH, et al. Pulmonary vein isolation for the maintenance of sinus rhythm in patients with atrial fibrillation: a meta-analysis of randomized, controlled trials. Circ Arrhythm Electrophysiol. 2009; 2:626-33. [PubMed: 20009077]

2. Cappato R, Calkins H, Chen SA, et al. Updated worldwide survey on the methods, efficacy, and safety of catheter ablation for human atrial fibrillation. Circ Arrhythm Electrophysiol. 2010; 3:32-8. [PubMed: 19995881]

3. Blomstrom, Lundqvist C.; Auricchio, A.; Brugada, J., et al. The use of imaging for electrophysiological and devices procedures: a report from the first European Heart Rhythm Association Policy Conference, jointly organized with the European Association of Cardiovascular Imaging (EACVI), the Council of Cardiovascular Imaging and the European Society of Cardiac Radiology. Europace. 2013; 15:927-36. [PubMed: 23729412]

4. Bunch TJ, Day JD. Examining the risks and benefits of transesophageal echocardiogram imaging during catheter ablation for atrial fibrillation. Circ Arrhythm Electrophysiol. 2012; 5:621-3. [PubMed: 22895599]

5. Ferguson JD, Helms A, Mangrum JM, et al. Catheter ablation of atrial fibrillation without fluoroscopy using intracardiac echocardiography and electroanatomic mapping. Circ Arrhythm Electrophysiol. 2009; 2:611-19. [PubMed: 20009075]

6. Hamdan A, Charalampos K, Roettgen R, et al. Magnetic resonance imaging versus computed tomography for characterization of pulmonary vein morphology before radiofrequency catheter ablation of atrial fibrillation. Am J Cardiol. 2009; 104:1540-6. [PubMed: 19932789]

7. Piccini JP, Sinner MF, Greiner MA, et al. Outcomes of medicare beneficiaries undergoing catheter ablation for atrial fibrillation. Circulation. 2012; 126:2200-7. [PubMed: 23019293]

8. Quan H, Sundararajan V, Halfon P, et al. Coding algorithms for defining comorbidities in ICD-9CM and ICD-10 administrative data. Med Care. 2005; 43:1130-9. [PubMed: 16224307]

9. Birman-Deych E, Radford MJ, Nilasena DS, et al. Use and effectiveness of warfarin in Medicare beneficiaries with atrial fibrillation. Stroke. 2006; 37:1070-4. [PubMed: 16528001]

10. Tirschwell DL, Longstreth WT Jr. Validating administrative data in stroke research. Stroke. 2002; 33:2465-70. [PubMed: 12364739]

11. Fisher ES, Wennberg DE, Stukel TA, et al. The implications of regional variations in Medicare spending. Part 1: the content, quality, and accessibility of care. Ann Intern Med. 2003; 138:27387. [PubMed: 12585825] 
12. Wallace TW, Atwater BD, Daubert JP, et al. Prevalence and clinical characteristics associated with left atrial appendage thrombus in fully anticoagulated patients undergoing catheter-directed atrial fibrillation ablation. J Cardiovasc Electrophysiol. 2010; 21:849-52. [PubMed: 20158561]

13. Calkins H, Kuck KH, Cappato R, et al. 2012 HRS/EHRA/ECAS Expert Consensus Statement on Catheter and Surgical Ablation of Atrial Fibrillation: Recommendations for Patient Selection, Procedural Techniques, Patient Management and Follow-up, Definitions, Endpoints, and Research Trial Design: A report of the Heart Rhythm Society (HRS) Task Force on Catheter and Surgical Ablation of Atrial Fibrillation. Heart Rhythm. 2012; 9:632-96. Developed in partnership with the European Heart Rhythm Association (EHRA), a registered branch of the European Society of Cardiology (ESC) and the European Cardiac Arrhythmia Society (ECAS); and in collaboration with the American College of Cardiology (ACC), American Heart Association (AHA), the Asia Pacific Heart Rhythm Society (APHRS), and the Society of Thoracic Surgeons (STS). Endorsed by the governing bodies of the American College of Cardiology Foundation, the American Heart Association, the European Cardiac Arrhythmia Society, the European Heart Rhythm Association, the Society of Thoracic Surgeons, the Asia Pacific Heart Rhythm Society, and the Heart Rhythm Society. e21. [PubMed: 22386883]

14. Martinek M, Nesser HJ, Aichinger J, et al. Impact of integration of multislice computed tomography imaging into three-dimensional electroanatomic mapping on clinical outcomes, safety, and efficacy using radiofrequency ablation for atrial fibrillation. Pacing Clin Electrophysiol. 2007; 30:1215-23. [PubMed: 17897124]

15. Caponi D, Corleto A, Scaglione M, et al. Ablation of atrial fibrillation: does the addition of threedimensional magnetic resonance imaging of the left atrium to electroanatomic mapping improve the clinical outcome?: a randomized comparison of Carto-Merge vs. Carto-XP three-dimensional mapping ablation in patients with paroxysmal and persistent atrial fibrillation. Europace. 2010; 12:1098-104. [PubMed: 20400766]

16. Carpen M, Matkins J, Syros G, et al. First experience of 3D rotational angiography fusion with NavX electroanatomical mapping to guide catheter ablation of atrial fibrillation. Heart Rhythm. 2013; 10:422-7. [PubMed: 23164737]

17. Reddy VY, Shah D, Kautzner J, et al. The relationship between contact force and clinical outcome during radiofrequency catheter ablation of atrial fibrillation in the TOCCATA study. Heart Rhythm. 2012; 9:1789-95. [PubMed: 22820056]

18. Neuzil P, Reddy VY, Kautzner J, et al. Electrical reconnection after pulmonary vein isolation is contingent on contact force during initial treatment: results from the EFFICAS I study. Circ Arrhythm Electrophysiol. 2013; 6:327-33. [PubMed: 23515263]

19. Brooks AG, Wilson L, Chia NH, et al. Accuracy and clinical outcomes of CT image integration with Carto-Sound compared to electro-anatomical mapping for atrial fibrillation ablation: a randomized controlled study. Int J Cardiol. 2013; 168:2774-82. [PubMed: 23623342] 


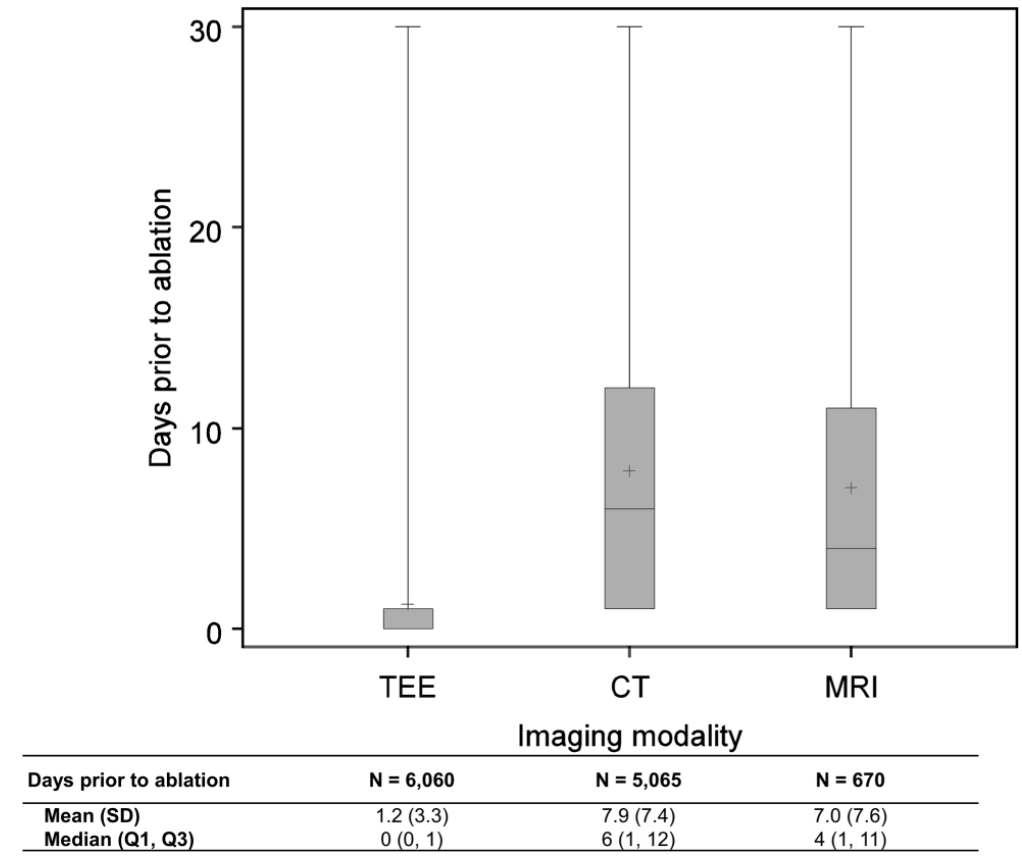

Figure 1.

Timing of preprocedural imaging before atrial fibrillation ablation. TEE, transoesophageal echocardiogram. 

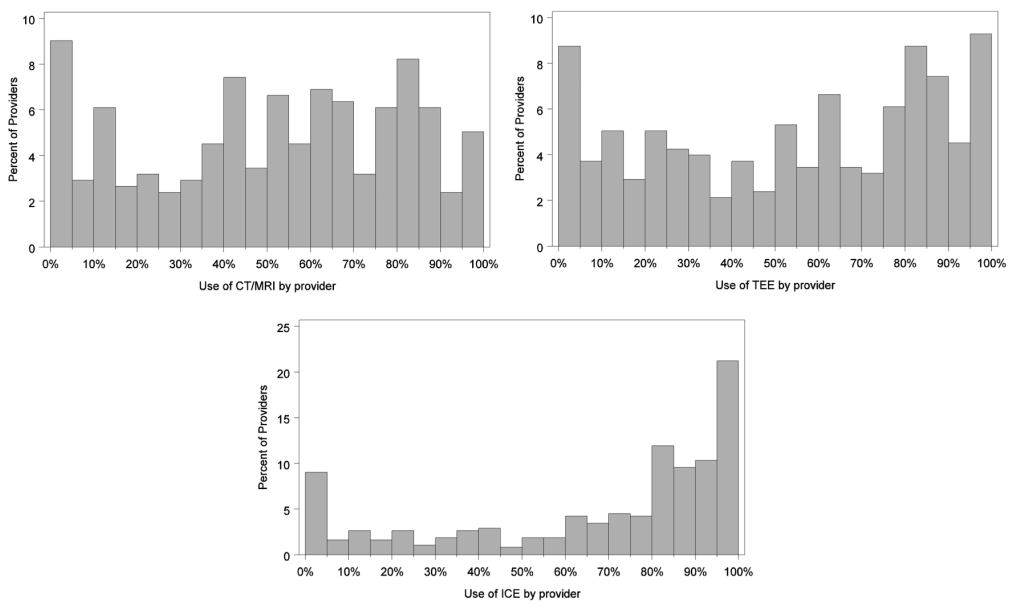

Figure 2.

Practice variation in utilisation of periprocedural imaging modalities. For each imaging modality (CT/MRI, TEE, ICE), the use of imaging is plotted on the x-axis (\% of ablations where the modality is used) and the proportion of practices using the modality at the corresponding rate is on the y-axis. Practices determined by 5-digit ZIP code; only those with $>5$ ablations in the study cohort were included here ( $n=337$ providers). TEE, transoesophageal echocardiogram; ICE, intracardiac echocardiography. 


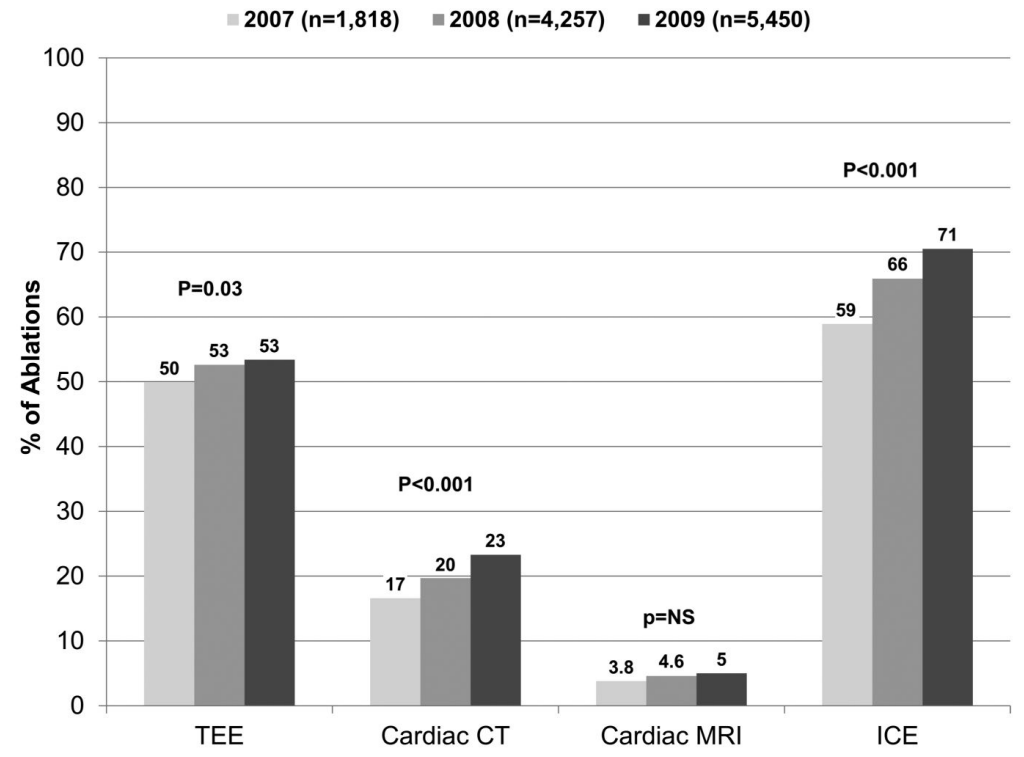

Figure 3.

Temporal trends in imaging utilisation before (CT, MRI, TEE) and during (ICE) atrial fibrillation ablation. TEE, transoesophageal echocardiogram; ICE, intracardiac echocardiography. 
Table 2

Baseline characteristics, stratified by periprocedural imaging modality

\begin{tabular}{|c|c|c|c|c|c|}
\hline & EAM only (n=3238) & EAM+TEE $(\mathrm{N}=2563)$ & EAM+CT/MRI (N=2227) & EAM+CT/MRI+TEE $(\mathrm{N}=3497)$ & p Value \\
\hline \multicolumn{6}{|l|}{ Other periprocedural imaging } \\
\hline TEE & - & $2563(100.0 \%)$ & - & $3497(100.0 \%)$ & $<0.001$ \\
\hline $\mathrm{CT}$ or MRI & - & - & $2227(100.0 \%)$ & $3497(100.0 \%)$ & $<0.001$ \\
\hline ICE & $1761(54.4 \%)$ & $1535(59.9 \%)$ & $1633(73.3 \%)$ & $2786(79.7 \%)$ & $<0.001$ \\
\hline Catheter-based mapping & $58(1.8 \%)$ & $33(1.3 \%)$ & $14(0.6 \%)$ & $28(0.8 \%)$ & $<0.001$ \\
\hline \multicolumn{6}{|l|}{ Demographics } \\
\hline Age, mean (SD) & $72.4(5.3)$ & $71.7(5.0)$ & $71.2(4.5)$ & $71.0(4.4)$ & $<0.001$ \\
\hline Male & $1899(58.6 \%)$ & $1612(62.9 \%)$ & $1230(55.2 \%)$ & $2085(59.6 \%)$ & $<0.001$ \\
\hline Race & & & & & $<0.001$ \\
\hline White & $3094(95.6 \%)$ & $2471(96.4 \%)$ & $2154(96.7 \%)$ & $3411(97.5 \%)$ & \\
\hline Black & $59(1.8 \%)$ & $47(1.8 \%)$ & $26(1.2 \%)$ & $23(0.7 \%)$ & \\
\hline Other/unknown & $85(2.6 \%)$ & $45(1.8 \%)$ & $47(2.1 \%)$ & $63(1.8 \%)$ & \\
\hline \multicolumn{6}{|l|}{ Comorbidities } \\
\hline Atrial flutter & $1404(43.4 \%)$ & $1086(42.4 \%)$ & $797(35.8 \%)$ & $1162(33.2 \%)$ & $<0.001$ \\
\hline Cancer & $360(11.1 \%)$ & $301(11.7 \%)$ & $251(11.3 \%)$ & $347(9.9 \%)$ & 0.12 \\
\hline Chronic kidney disease & $277(8.6 \%)$ & $231(9.0 \%)$ & $120(5.4 \%)$ & $202(5.8 \%)$ & $<0.001$ \\
\hline Chronic pulmonary disease & $766(23.7 \%)$ & $647(25.2 \%)$ & $526(23.6 \%)$ & $845(24.2 \%)$ & 0.48 \\
\hline Dementia & $19(0.6 \%)$ & $13(0.5 \%)$ & $13(0.6 \%)$ & $*$ & 0.25 \\
\hline Diabetes mellitus & $759(23.4 \%)$ & $613(23.9 \%)$ & $467(21.0 \%)$ & $737(21.1 \%)$ & 0.009 \\
\hline Heart failure & $855(26.4 \%)$ & $745(29.1 \%)$ & $512(23.0 \%)$ & $887(25.4 \%)$ & $<0.001$ \\
\hline Hypertension & $2583(79.8 \%)$ & $2073(80.9 \%)$ & $1768(79.4 \%)$ & $2836(81.1 \%)$ & 0.30 \\
\hline Ischaemic heart disease & $1666(51.5 \%)$ & $1414(55.2 \%)$ & $1180(53.0 \%)$ & $1871(53.5 \%)$ & 0.04 \\
\hline Peripheral vascular disease & $506(15.6 \%)$ & $455(17.8 \%)$ & $373(16.7 \%)$ & $668(19.1 \%)$ & 0.002 \\
\hline Stroke/TIA & $240(7.4 \%)$ & $234(9.1 \%)$ & $166(7.5 \%)$ & $281(8.0 \%)$ & 0.08 \\
\hline Valvular heart disease & $1181(36.5 \%)$ & $1441(56.2 \%)$ & $834(37.4 \%)$ & $1889(54.0 \%)$ & $<0.001$ \\
\hline $\mathrm{CHADS}_{2}$ score & & & & & $<0.001$ \\
\hline 0 & $390(12.0 \%)$ & $274(10.7 \%)$ & $293(13.2 \%)$ & $384(11.0 \%)$ & \\
\hline 1 & $1108(34.2 \%)$ & $927(36.2 \%)$ & $846(38.0 \%)$ & $1394(39.9 \%)$ & \\
\hline 2 & $991(30.6 \%)$ & $752(29.3 \%)$ & $669(30.0 \%)$ & $1049(30.0 \%)$ & \\
\hline 3 & $501(15.5 \%)$ & $380(14.8 \%)$ & $291(13.1 \%)$ & $451(12.9 \%)$ & \\
\hline 4 & $181(5.6 \%)$ & $155(6.0 \%)$ & $92(4.1 \%)$ & $170(4.9 \%)$ & \\
\hline 5 & $51(1.6 \%)$ & $60(2.3 \%)$ & $31(1.4 \%)$ & $38(1.1 \%)$ & \\
\hline 6 & $16(0.5 \%)$ & $15(0.6 \%)$ & $*$ & $11(0.3 \%)$ & \\
\hline $\mathrm{CHADS}_{2}$ score, $\geq 2$ & $1740(53.7 \%)$ & $1362(53.1 \%)$ & $1088(48.9 \%)$ & $1719(49.2 \%)$ & $<0.001$ \\
\hline \multicolumn{6}{|l|}{ INR testing } \\
\hline Within 30 days prior & $2474(76.4 \%)$ & $2009(78.4 \%)$ & $1821(81.8 \%)$ & $2943(84.2 \%)$ & $<0.001$ \\
\hline Within 30 days after & $2658(82.1 \%)$ & $2268(88.5 \%)$ & $1976(88.7 \%)$ & $3201(91.5 \%)$ & $<0.001$ \\
\hline Geographic location ${ }^{\dagger}$ & & & & & $<0.001$ \\
\hline Northeast & $318(9.8 \%)$ & $269(10.5 \%)$ & $281(12.6 \%)$ & $497(14.2 \%)$ & \\
\hline
\end{tabular}




\begin{tabular}{lccccc}
\hline & EAM only $(\mathbf{n}=\mathbf{3 2 3 8})$ & EAM+TEE $(\mathbf{N = 2 5 6 3})$ & EAM+CT/MRI (N=2227) & EAM+CT/MRI+TEE (N=3497) & p Value \\
\hline South & $1185(36.6 \%)$ & $1336(52.1 \%)$ & $758(34.0 \%)$ & $1462(41.8 \%)$ \\
Midwest & $742(22.9 \%)$ & $472(18.4 \%)$ & $539(24.2 \%)$ & $914(26.1 \%)$ \\
West & $993(30.7 \%)$ & $486(19.0 \%)$ & $649(29.1 \%)$ & $624(17.8 \%)$ \\
Inpatient procedure & $1748(54.0 \%)$ & $1493(58.3 \%)$ & $1269(57.0 \%)$ & $2083(59.6 \%)$ & $<0.001$ \\
\hline
\end{tabular}

EAM, electroanatomic mapping; ICE: intracardiac echocardiography; INR, international normalised ratio; TEE, transoesophageal echocardiogram; TIA, transient ischaemic attack.

* 10 or fewer patients in this cell.

${ }^{\dagger}$ US regions, by state. 
Table 3

Unadjusted 6-month outcomes, stratified by periprocedural imaging strategy

\begin{tabular}{llllll}
\hline Outcome & EAM only $(\mathbf{n = 3 2 3 8})$ & EAM+TEE $(\mathbf{N = 2 5 6 3 )}$ & EAM+CT/MRI (N=2227) & EAM+CT/MRI+TEE (N=3497) & p Value \\
\hline $\begin{array}{l}\text { Mortality } \\
\begin{array}{l}\text { Composite, overall } \\
\text { (excluding mortality) }\end{array}\end{array}$ & $54(1.9 \%)$ & $37(1.6 \%)$ & $23(1.1 \%)$ & $32(1.0 \%)$ & 0.03 \\
$\quad 138(4.5 \%)$ & $121(5.1 \%)$ & $71(3.4 \%)$ & $123(3.8 \%)$ & 0.02 \\
$\quad$ Stroke/TIA & $30(1.0 \%)$ & $19(0.8 \%)$ & $*$ & $14(0.4 \%)$ & 0.009 \\
$\quad$ Severe bleeding ${ }^{\ddagger}$ & $27(0.8 \%)$ & $28(1.1 \%)$ & $24(1.1 \%)$ & $31(0.9 \%)$ & 0.67 \\
$\quad$ Repeat ablation & $177(6.2 \%)$ & $148(6.7 \%)$ & $154(7.9 \%)$ & $177(6.0 \%)$ & 0.03 \\
Composite, cardiovascular $\$$ & $114(3.8 \%)$ & $96(4.1 \%)$ & $50(2.4 \%)$ & $93(2.9 \%)$ & 0.005 \\
\hline
\end{tabular}

EAM, electroanatomic mapping; TEE, transoesophageal echocardiogram; TIA, transient ischaemic attack.

* 10 or fewer patients in this cell

${ }^{\dagger}$ Includes stroke/TIA, haemopericardium, cardiac tamponade, heart failure and vascular complications requiring surgery.

*Includes haemopericardium, cardiac tamponade and vascular complications requiring surgery.

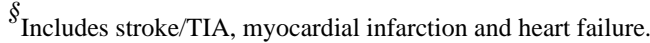




\section{Table 4}

Adjusted associations between periprocedural imaging and outcomes

\begin{tabular}{|c|c|c|c|c|c|c|}
\hline & \multicolumn{2}{|l|}{ TEE } & \multicolumn{2}{|l|}{ CT/MRI } & \multicolumn{2}{|l|}{ ICE } \\
\hline & HR $(95 \%$ CI $)$ & p Value & HR $(95 \%$ CI $)$ & p Value & HR $(95 \%$ CI $)$ & p Value \\
\hline Mortality & $0.92(0.64$ to 1.30$)$ & 0.62 & 0.85 (0.59 to 1.23$)$ & 0.39 & $0.78(0.54$ to 1.13$)$ & 0.19 \\
\hline Composite, overall(excluding mortality) ${ }^{*}$ & 1.04 (0.86 to 1.27$)$ & 0.66 & $0.84(0.68$ to 1.02$)$ & 0.08 & $1.08(0.88$ to 1.34$)$ & 0.45 \\
\hline Stroke/TIA & $0.94(0.58$ to 1.53$)$ & 0.80 & $0.46(0.27$ to 0.78$)$ & 0.004 & $0.91(0.55$ to 1.51$)$ & 0.71 \\
\hline Severe bleeding ${ }^{\dagger}$ & $0.98(0.66$ to 1.45$)$ & 0.90 & $0.94(0.63$ to 1.39$)$ & 0.76 & $1.78(1.11$ to 2.83$)$ & 0.02 \\
\hline Repeat ablation & $0.90(0.76$ to 1.07$)$ & 0.25 & $1.16(0.98$ to 1.37$)$ & 0.09 & $0.68(0.57$ to 0.82$)$ & $<0.001$ \\
\hline Composite, cardiovascular ${ }^{*}$ & $1.05(0.84$ to 1.31$)$ & 0.66 & $0.79(0.63$ to 1.00$)$ & 0.048 & $0.99(0.78$ to 1.25$)$ & 0.91 \\
\hline
\end{tabular}

ICE, intracardiac echocardiography; TEE, transoesophageal echocardiogram; TIA, transient ischaemic attack.

Includes stroke/TIA, haemopericardium, cardiac tamponade, heart failure and vascular complications requiring surgery.

${ }^{\dagger}$ Includes haemopericardium, cardiac tamponade and vascular complications requiring surgery.

*Includes stroke/TIA and heart failure. 